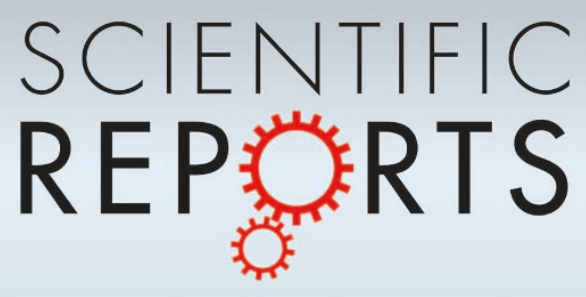

OPEN

SUBJECT AREAS:

NANOBIOTECHNOLOGY

NANOTECHNOLOGY

SURFACE PATTERNING AND

IMAGING

DNA

Received

30 May 2012

Accepted

20 July 2012

Published

7 August 2012

Correspondence and requests for materials should be addressed to

T.S. (tsen@uclan.ac. uk) or I.J.B. (i.j.bruce@ kent.ac.uk)

* Current Address:

Surface Patterning

Group, Institute of

Nanotechnology and

Bio-engineering,

Centre for Materials

Science, School of

Forensic and

Investigative Sciences,

University of Central

Lancashire, Preston,

PR 1 2HE, United

Kingdom.

\section{Surface engineering of nanoparticles in suspension for particle based bio-sensing}

Tapas Sen* \& lan J. Bruce

Nanobiotechnology Research Group, School of Biosciences, University of Kent, Canterbury, CT2 7NJ, United Kingdom.

Surface activation of nanoparticles in suspension using amino organosilane has been carried out via strict control of a particle surface ad-layer of water using a simple but efficient protocol 'Tri-phasic Reverse Emulsion' (TPRE). This approach produced thin and ordered layers of particle surface functional groups which allowed the efficient conjugation of biomolecules. When used in bio-sensing applications, the resultant conjugates were highly efficient in the hybrid capture of complementary oligonucleotides and the detection of food borne microorganism. TPRE overcomes a number of fundamental problems associated with the surface modification of particles in aqueous suspension viz. particle aggregation, density and organization of resultant surface functional groups by controlling surface condensation of the aminosilane. The approach has potential for application in areas as diverse as nanomedicine, to food technology and industrial catalysis.

M aterials surface patterning with chemicals and bio-chemicals at the nanometer length scale has a fundamental importance ${ }^{1,2}$ in the fabrication and application of nano-devices ${ }^{3,4}$ as such materials utilities are thought to be directly related to the density and orientation of their surface functional groups. Organosilanes are particularly important molecules in that context. Surface patterning on flat surfaces (e.g. silicon wafers, diamond or quartz) is a relatively simple process and can be defined as two dimensional (XY) where chemicals or bio-chemicals interact perpendicular to the XY plane through the Z axis (see Figure S1). Recently S. De Feyter has also reported ${ }^{5}$ surface patterning in a three dimensional context. However, surface patterning of nanoparticles in suspension can be a complex process due to the potential for particle aggregation, Brownian motion, solvent effects etc. Efficient surface patterning using aminosilanes has been reported ${ }^{6-8}$ on flat surfaces where the presence of water within the system was strictly controlled so as permit the formation of a surface silane monolayer. The density of such a monolayer has been reported as 5.3 molecules $/ \mathrm{nm}^{2}$ and the thickness as $0.7 \mathrm{~nm}$. Recently ${ }^{9}$ the same concept has been applied to mesoporous solids with controlled amounts of intra-pore water where confocal laser scanning microscopy was used to monitor the effect of water on the amino functionalisation of pore surfaces. The surface patterning/engineering of mesoporous solids with organic frameworks has also been investigated ${ }^{10}$. In the case of nanoparticles dispersed in aqueous suspension, surface patterning with aminosilanes can be a very complex process due to the various possible reactions of such molecules with water (see Figure S2 and text). The rate constants $\left(\mathrm{k}_{1}, \mathrm{k}_{2}, \mathrm{k}_{3}, \mathrm{k}_{4}, \mathrm{k}_{4}{ }^{\prime}, \mathrm{k}_{5}\right)$ of those various reactions effectively control the aminosilane patterning of particles' surfaces. In order to produce a uniform surface monolayer of $-\mathrm{NH}_{2}$, ideally $\mathrm{k}_{1}$ should be equal to $\mathrm{k}_{4}$ and the other rate constants $\left(\mathrm{k}_{2}, \mathrm{k}_{3}, \mathrm{k}_{4}{ }^{\prime}\right.$ and $\left.\mathrm{k}_{5}\right)$ should be zero. However, we have reported that aminosilane surface patterning of nanoparticles in water produces amine groups which are mostly internalized or sequestered ${ }^{11,12}$. Surface functionalisation of nanoparticles has recently been reviewed ${ }^{13}$ for bio-medical applications however, the effect of water on (i) surface activation and consequently (ii) surface functionalisation/ conjugation with biomolecules and (iii) the efficiency with which the conjugates function in applications has not been investigated or reported previously. In fact the formation of ordered surface amine groups on nanoparticles in aqueous suspension during aminosilanisation remains as a challenge in the fabrication of functionalised nanoparticulates for highly specific and targeted applications from food technology ${ }^{14,15}$ to nanomedicine ${ }^{16}$ and industrial catalysis ${ }^{17}$.

Herein, we report the surface engineering of nanoparticles in suspension by strict control of a water layer surrounding the nanoparticles via TPRE and the materials surface functionalisation with oligonucleotide bioligands for the efficient detection of food born microorganisms as a particle based bio-sensor. TPRE is so called as it involves the nanoparticles (the first, solid phase), a thin water ad-layer (the second phase) and an organic nonwater miscible third phase (toluene). It is a reverse phase as the water-coated nanoparticles are suspended in the organic solvent (demonstrated in Figure S3). The method produces an ordered layer of surface amine groups on the nanoparticles and their oligonucleotide conjugated forms have been demonstrated to possess exceptionally 
high efficiency (92\%) in hybrid capture of complementary nucleic acids. When used to detect food borne microorganisms, such material exhibited both high sensitivity and selectivity in identifying and discriminating specific target DNA sequences from complex mixtures of DNAs derived from pathogenic and spoilage bacteria in food.

\section{Results}

In TPRE, aminopropyltriethoxy silane was added to a tri-phasic reverse emulsion (see Figure 1) prepared by separating bulk water either magnetically (silica coated core-shell superparamagnetic iron oxide nanoparticles) or by centrifugation (diamagnetic silica nanoparticles) from particle suspensions followed by their re-dispersion in toluene in the presence of a biocompatible surfactant Triton X100 (movie S1; Supplementary Information and Methods). Amino organosilane molecules are soluble but do not hydrolyse or self- condense in toluene hence they remain un-reacted in the continuous toluene phase. They can only hydrolyse and subsequently condense where water is present in the system which in TPRE is as a surface ad-layer on the nanoparticles (see Figure 1 and movie S1). This water layer is chemi- and physisorbed to the particles surface and does not diffuse into the organic solvent (toluene) due to that phase's hydrophobic nature. In TPRE, problems relating to particle aggregation and selfcondensation of aminosilane monomers to oligomeric and polymeric forms (see Figure S3b) in the solution phase are eliminated.

Material physical and chemical characterization. Size, surface area and surface amine density. Silica coated spherical core-shell superparamagnetic nanoparticles were measured to be approximately $40 \mathrm{~nm}$ in diameter (see Figure S4a) and diamagnetic silica spheres approximately $400 \mathrm{~nm}$ in diameter (see Figure S4b). Surface areas of the nanoparticles were determined by Brunauer, Emmett, Teller (BET) nitrogen adsorption and used in the calculation of surface functional group density $/ \mathrm{nm}^{2}$. The BET surface area of the superparamagnetic nanoparticles was observed to be $30 \mathrm{~m}^{2} / \mathrm{g}$. The amounts of surface and total amine of aminosilanised materials were determined colorimetrically ${ }^{18}$ and by combustion $(\mathrm{CHN})$ analysis ${ }^{11,12}$ respectively and surface amine density ( $\mathrm{nmol} / \mathrm{mg}$ or molecules $/ \mathrm{nm}^{2}$ ) was calculated by integrating the amine values obtained from a fixed mass of material with the particles' surface areas.

Aminosilanised superparamagnetic core-shell nanoparticles prepared via TPRE were observed to posses almost all their $-\mathrm{NH}_{2}$ groups at the surface (approximately 85\%) with a small amount in a sequestered or internal state (Figure 2a). By comparison when aminosilanisation was carried out in water, the materials were observed to possess only $21 \%$ of their amine at the surface with the remainder as sequestered. The surface amine density of silica-magnetic nanoparticles aminosilanised via TPRE was measured to be $240 \mathrm{nmol} / \mathrm{mg}$ (calculated to be 4.8 molecules $/ \mathrm{nm}^{2}$ ) compared to 0.5 molecules $/ \mathrm{nm}^{2}$ for those silanised in water. The high ratio of surface to total amine density (85\%) obtained for TPRE materials is direct proof of its control over the surface condensation of aminosilane and the value of amine density observed was very close to that reported for a monolayer on flat surfaces ${ }^{6,7}$. Ellipsometry has been used by various researchers to determine the monolayer thickness of aminosilane on flat surfaces ${ }^{6,7}$. The thickness value was measured to be $0.7 \mathrm{~nm}$ on silicon wafers ${ }^{6,7}$, but the technique was not used in our experiments as its application to particulates can be unreliable due to the presence of solvent in suspension. Rather we have verified particle size (diameter) before and after aminosilanisation using a particle size analyser (Malvern Zetasizer). No change in nanoparticle diameters (see Figure S5) were observed for TPRE aminosilanised material (the particle size analyser possesses a measurement tolerance limit of $1 \mathrm{~nm})$. No observed change in particle size was an indication that no particle aggregation or significant siloxane precipitation on the nanoparticles surfaces during TPRE had occurred. However a clear shift in the particle size was observed when particle aminosilanisation was carried out in water. The structural nature of the surface aminosilane layer was verified by solid state NMR.

${ }^{29}$ Si single pulse (SP) and ${ }^{29} \mathrm{Si}^{1}{ }^{1} \mathrm{H}$ cross polarization $(\mathrm{CP})$ magic angle sample spinning NMR. Solid state nuclear magnetic resonance (NMR) can be a powerful tool for elucidating the molecular structure of materials such as those described in Figure 1 but superparamagnetic material has a paramagnetic broadening effect on NMR. Consequently diamagnetic silica nanoparticles were used as a model in these experiments. After aminosilanisation by TPRE and in water the surface amine densities of the silica nanoparticles were measured to be 4.8 and 0.9 molecules $/ \mathrm{nm}^{2}$ respectively indicating that they had behaved in an effectively identical manner to the silica-magnetite nanoparticles. Figure 3 presents the ${ }^{29} \mathrm{Si}$ single pulse (SP) and ${ }^{29} \mathrm{Si}^{-1} \mathrm{H}$ cross polarisation (CP) magic angle sample spinning (MAS) solid state NMR spectra of TPRE aminosilanised diamagnetic silica nanoparticles along with three 'reference samples' (i) non-aminosilanised silica nanoparticles, (ii) silica obtained directly from aminopropyltriethoxy silane (APTS) condensation/polymerisation in water and (iii) diamagnetic silica nanoparticles aminosilanised in water. The non-aminosilanised silica spheres exhibited peaks (Figure 3a) in the region of -90 to $-120 \mathrm{ppm}$ indicating the presence of only $\mathrm{Q}^{\mathrm{n}}(\mathrm{Si})$ species in both SP and CP mode. Peak intensities support the observation that relative concentrations followed the trend $\left(\mathrm{Q}^{4}>\mathrm{Q}^{3}>\mathrm{Q}^{2}\right.$ Si-species) in SP mode but the intensity of the $\mathrm{Q}^{3}(\mathrm{Si})$ species was observed to be greatest in CP mode. This may be expected as $\mathrm{Q}^{3}$ and $\mathrm{Q}^{2}$ are capable of cross-polarisation transfer from neighboring protons whereas $\mathrm{Q}^{4}$ is not. However, this observation also suggests that the proportion of $\mathrm{Q}^{3}$ species is much higher than $\mathrm{Q}^{2}$ species, particularly as the density of neighbouring protons in $\mathrm{Q}^{2}$ is twice that in $\mathrm{Q}^{3}$. Material obtained from condensing/polymerising APTS in water exhibited no peaks (Figure $3 \mathrm{~b}$ ) in the region of -90 to $-120 \mathrm{ppm}$ indicating the absence of $\mathrm{Q}^{\mathrm{n}}(\mathrm{Si})$ species, however $\mathrm{T}^{\mathrm{n}}(\mathrm{Si})$ species were observed as a consequence of the presence of the Si bonded propyl

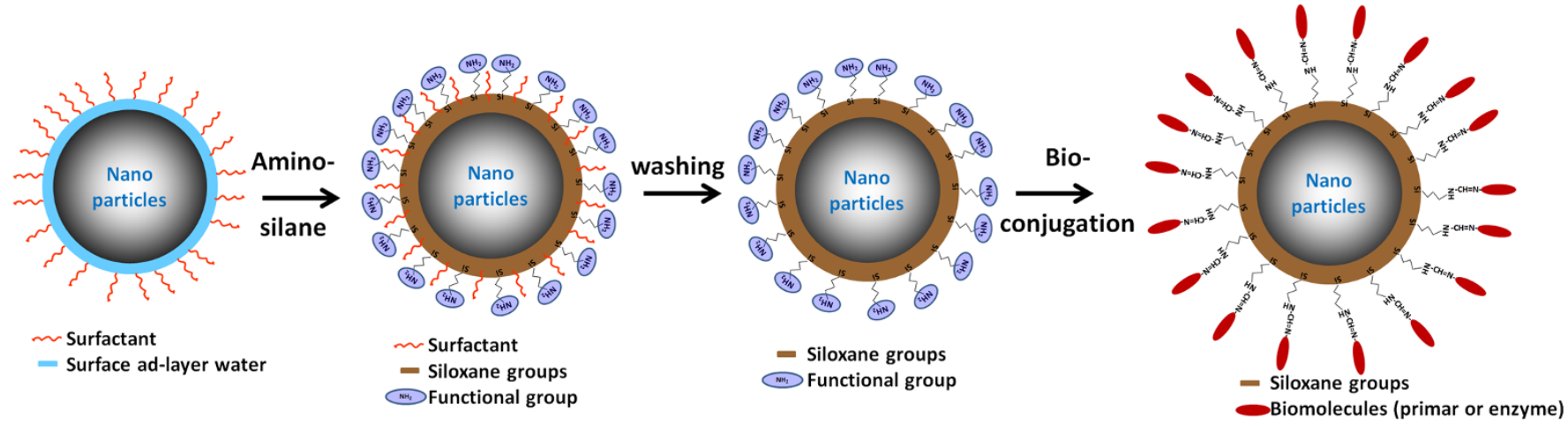

Figure $1 \mid$ A schematic diagram of TPRE for surface patterning of nanoparticles in suspension. 

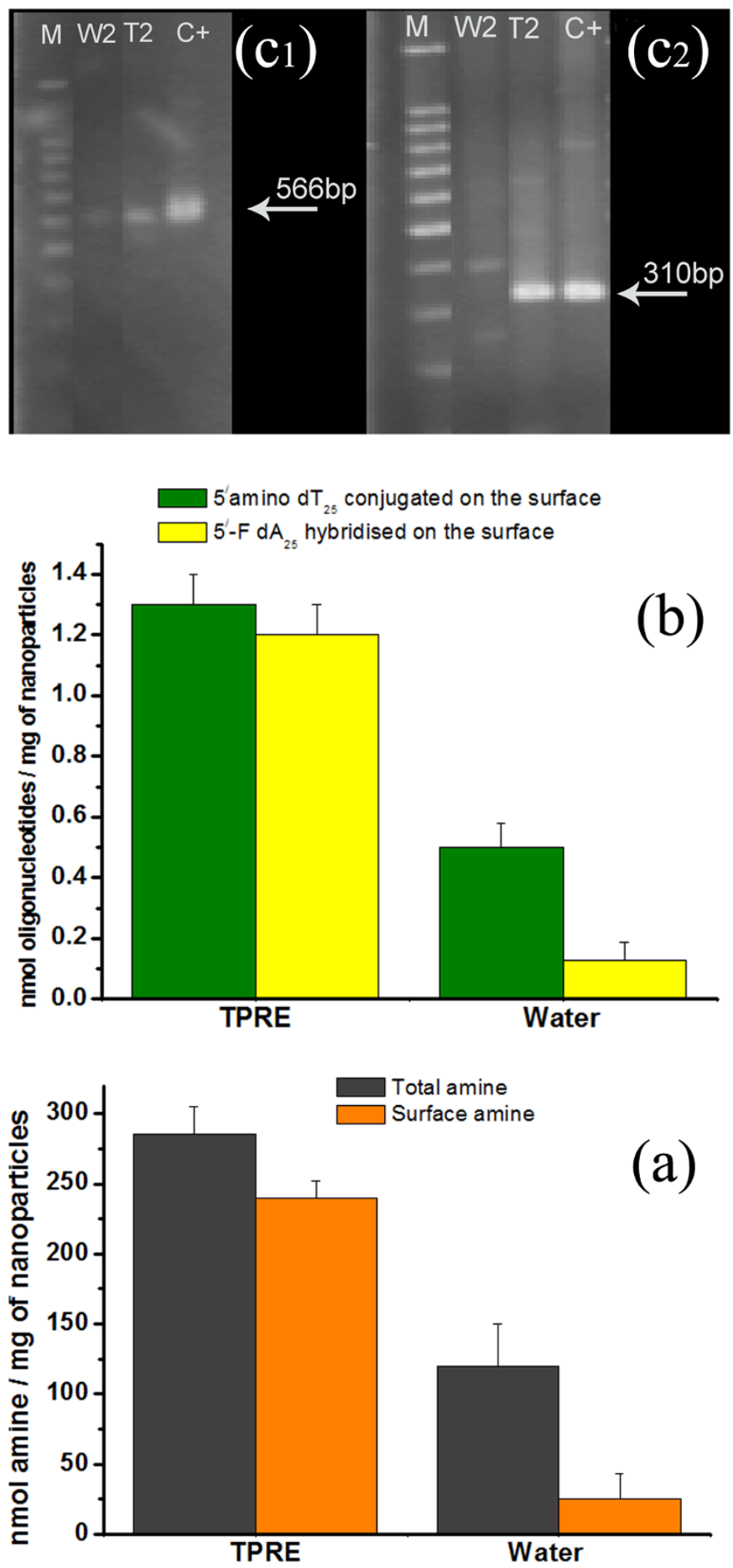

Figure $2 \mid$ Total and surface amine densities of nanoparticles aminosilanised via TPRE and in water. (a); $5^{\prime}-\mathrm{NH}_{2} \mathrm{dC}_{6} \mathrm{dT}_{25}$ oligonucleotide grafted and hybridized $5^{\prime} \mathrm{FdA}_{25}$ complementary oligonucleotide (b); hybrid capture using bacterial genomic DNA. PCR amplification of $566 \mathrm{bp}$ bacterial $16 \mathrm{~S}$ rRNA gene fragment (c1) and $310 \mathrm{bp}$ $p f k B-t h r S$ intergenic region from Salmonella enteric (c2). Abbreviations: M, 100 bp DNA molecular weight ladder (New England Biolabs Inc); W silica-magnetic nanoparticles aminosilanised in water and $\mathrm{T}$ aminosilanised silica-magnetite nanoparticles produced via TPRE; 1 and 2 correspond to 16S rRNA and Salmonella enterica DNA sequences, respectively; $\mathrm{C}+$, positive $\mathrm{PCR}$ control. amine group. Three different $\mathrm{T}^{\mathrm{n}}(\mathrm{Si})$ sites were observed with relative concentrations of $\mathrm{T}^{3}>\mathrm{T}^{2}>\mathrm{T}^{1}(\mathrm{Si})$ in SP mode but only two types of $\mathrm{T}$ sites were observed in CP mode. TPRE aminosilanised nanoparticles exhibited two types ( $\mathrm{T}$ and $\mathrm{Q}$ ) of Si environments (Figure 3d). Peak intensities support the supposition that concentrations of $\mathrm{T}$ sites in TPRE activated materials were higher than that for silica nanoparticles aminosilanised in water. This is consistent with the data obtained from combustion analysis i.e. the number of propyl amine groups based on $\mathrm{CHN}$ data. SP mode intensities of Si resonances in $\mathrm{T}$ sites were poor, however, cross-polarisation provided the necessary enhancement to signal as a consequence of spinpopulation energy transfer from neighbouring proton nuclei. Only $\mathrm{T}^{3}(\mathrm{Si})$ sites were observed in TPRE materials (Figure $3 \mathrm{~d}$ ) but both $\mathrm{T}^{2}$ and $\mathrm{T}^{3}(\mathrm{Si})$ sites were detected (Figure $3 \mathrm{c}$ ) in nanoparticles aminosilanised in water indicating two types of $\mathrm{T}(\mathrm{Si})$ environments. It was also observed that intensities of $\mathrm{Q}^{2}(\mathrm{Si})$ species were reduced in both amino-functionalised materials; most likely due to the loss of $\mathrm{Si}-\mathrm{OH}$ groups by conjugation with aminosilane via dehydration. The data obtained from material aminosilanised in water (i.e. the uncontrolled system) supports a previous report by Vanblaaderen et al ${ }^{19}$ who observed that amine groups can be $\mathrm{T}^{1}(\mathrm{Si})$ to $\mathrm{T}^{3}(\mathrm{Si})$ types consequent from the uncontrolled surface condensation of aminosilane (see Figure S3b) occurring in this scenario. From these data it is also reasonable to suggest that the relative increase in concentration of $\mathrm{Q}^{4}(\mathrm{Si})$ compared to $\mathrm{Q}^{3}(\mathrm{Si})$ and $\mathrm{Q}^{2}(\mathrm{Si})$ sites for aminosilanised nanoparticles when compared to non-aminosilanised silica nanoparticles resulted from the transformation of $\mathrm{Q}^{2}(\mathrm{Si})$ to $\mathrm{Q}^{3}(\mathrm{Si})$ to $\mathrm{Q}^{4}(\mathrm{Si})$ by condensation of aminosilane to particle surface. The NMR data clearly indicate a single $\mathrm{T}(\mathrm{Si})$ site formation resulting from aminosilanisation via TPRE strongly supporting the ordering of surface siliane groups on the nanoparticles illustrated in figure 1 .

${ }^{13} \mathrm{C}^{-1} \mathrm{H}$ cross polarization (CP) magic angle sample spinning (MAS) $N M R$. We have also verified the complete removal of surfactant (Triton X100) from the TPRE aminosilanised materials after washing in coupling solution by ${ }^{13} \mathrm{C}-{ }^{1} \mathrm{H}$ CPMAS NMR (see Figure 4). Three distinct regions labeled as $\mathrm{a}, \mathrm{b}$ and $\mathrm{c}$ were observed in the spectra of the various materials analysed. The three peaks visible in region 'a' correspond to the three carbon atoms of the amino propyl group $^{19}$ and non-aminosilanised diamagnetic silica nanoparticles exhibited no peaks in this region (black trace) as expected. Both condensed/polymerised APTS (blue trace) and APTS silanised silica nanoparticles (washed-green and unwashed-red) exhibited peaks in region 'a' due to their possession of aminopropyl groups. A small shift in the peak positions in region 'a' was observed for the sample aminosilanised by TPRE compared to condensed APTS suggesting that the carbon atoms of the propyl group were in a slightly different configuration in the two materials, perhaps due to an increase in ordering in the case of TPRE (confirmed by ${ }^{29} \mathrm{Si}$ MAS NMR). The multiple peaks observed in region 'b' correspond to the presence of various carbon atoms possessed by Triton X100 and were observed only in the case of unwashed samples of nanoparticles aminosilanised by TPRE. The absence of such peaks in region ' $b$ ' in the other samples clearly indicates the absence of Triton X100. Multiple peaks observed in region ' $c$ ' correspond to materials used in the construction and packing (tissue paper) of the NMR rotor. The latter was employed so as to achieve the required spinning speed for magic angle sample spinning experiments. All samples exhibited peaks in region ' $c$ ' and variations in their intensities were most likely due to the differences in the mass of tissue paper used for packing the rotor. These results confirm that no surfactant remained after washing of TPRE aminosilanised nanoparticles.

Biological application data. The ability of the nanoparticles' surface amino groups to be conjugated to bio-ligands and subsequently used in practical applications was tested by applying them in a molecular diagnostic/bio-sensing context. Firstly, the surface of 


\section{SP MAS}

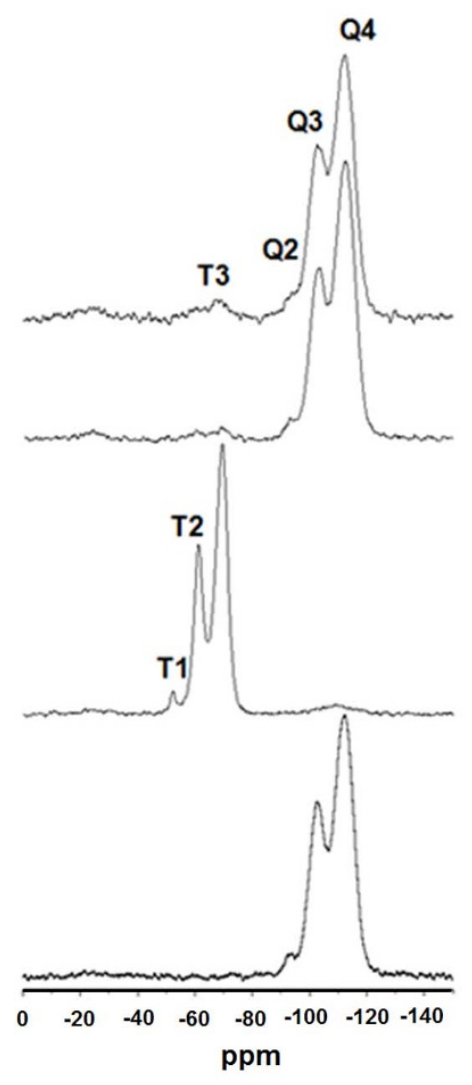

(a)
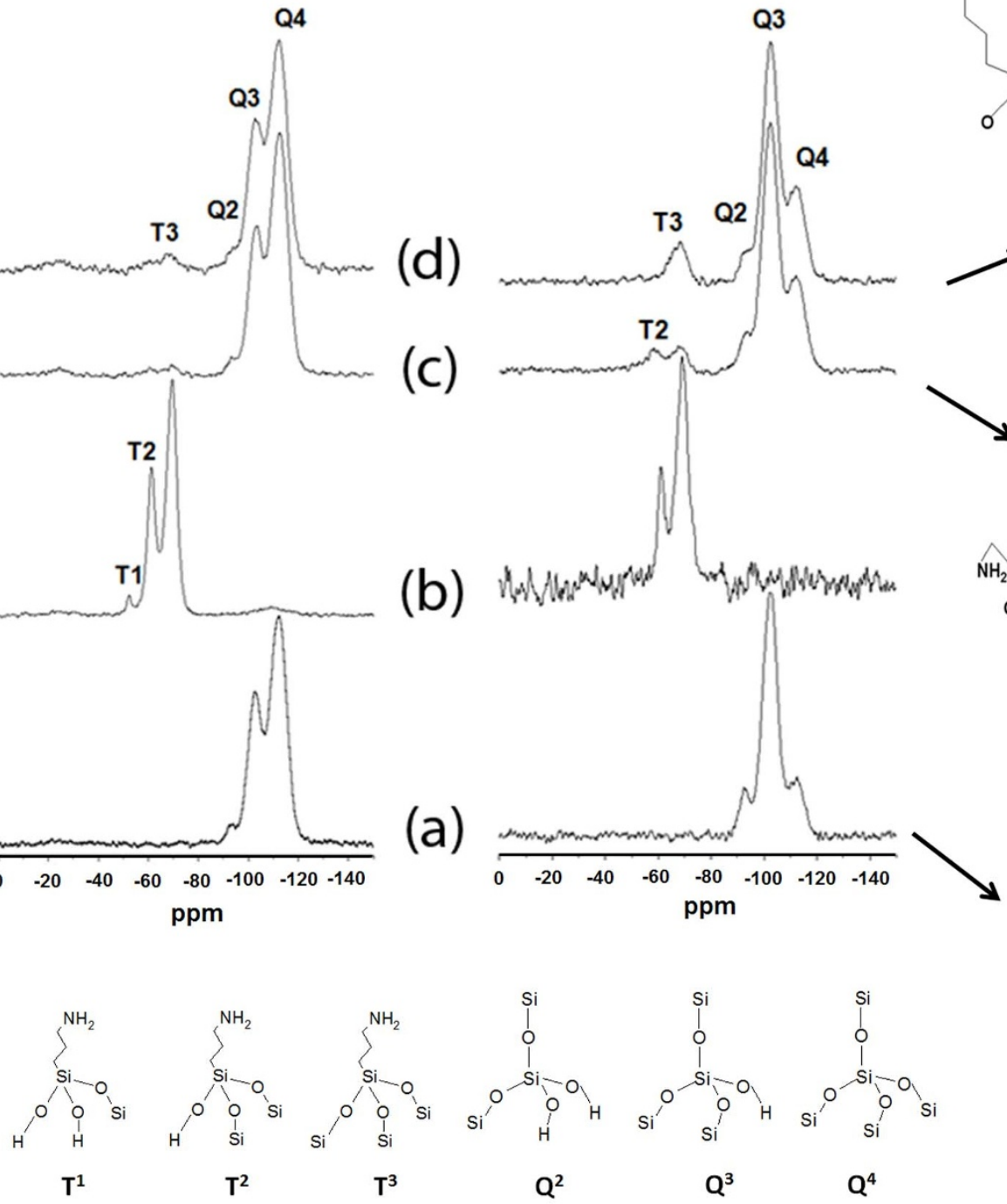

$\mathrm{Q}^{4}$
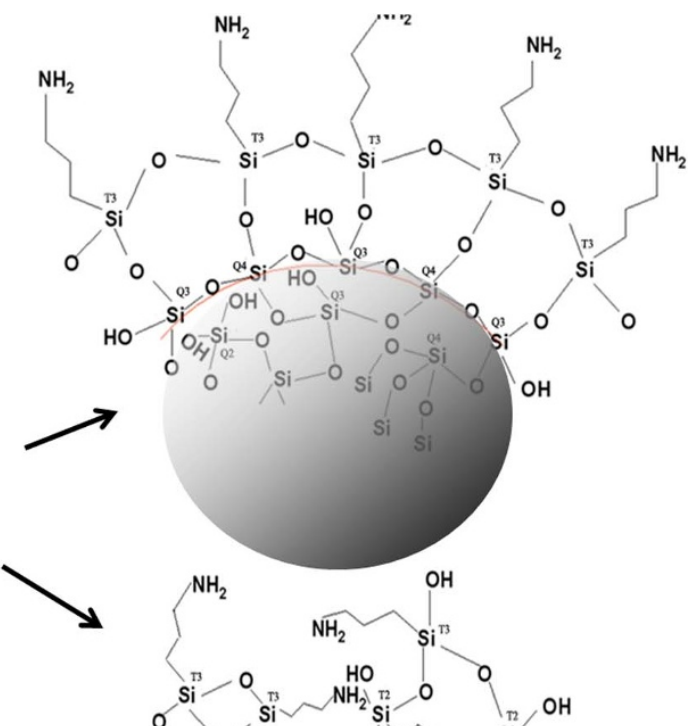

Figure $3{ }^{29} \mathrm{Si}$ single pulse (SP) and ${ }^{29} \mathrm{Si}-{ }^{-1} \mathrm{H}$ cross polarization (CP) magic angle sample spinning (MAS) spectra of non-aminosilanised diamagnetic silica nanoparticles. (a); condensed/polymerised APTS (b); silica nanoparticles aminosilanised in water (c) and silica nanoparticles aminosilanised via TPRE (d).

silica-magnetite nanoparticles aminosilanised via TPRE and water were conjugated with oligonucleotide $\left(5^{\prime} \mathrm{NH}_{2} \mathrm{dC}_{6} \mathrm{dT}_{25}\right)$ and the conjugates tested for their abilities to capture complementary target DNA $\left(5^{\prime} \mathrm{FdA}_{25}\right)$ in hybrid capture experiments (see Figure $\mathrm{S} 6$ in the supplementary information and Methods). Nanoparticles aminosilanised via TPRE were observed to exhibit a 92\% capture efficiency $(1.2 \mathrm{nmol} / \mathrm{mg})$ of $5^{\prime} \mathrm{FdA}_{25}$ oligonucleotide compared to $26 \%$ $(0.13 \mathrm{nmol} / \mathrm{mg})$ for those aminosilanised in water (see Figure $2 \mathrm{~b}$ ). In absolute terms of amount of oligonucleotide captured, this represented a difference of almost a nanomole of oligonucleotide per milligram of nanoparticles which is most likely to have resulted from an increased order in surface patterning of the oligonucleotide in the case of those particles activated via TPRE. This high level of hybridisation efficiency is a possible indication of improved accessibility to the grafted oligonucleotide $\left(5^{\prime} \mathrm{NH}_{2} \mathrm{dC}_{6} \mathrm{dT}_{25}\right)$ by the $5^{\prime} \mathrm{FdA}_{25}$ as a consequence of its surface ordering as presented in Figure 1.

A similar approach was then applied to generate oligonucleotide surface conjugated nanoparticles which were capable of recognising target sequences (see Methods) possessed by spoilage and pathogenic bacteria which can be found in the food. The hybrid captured target sequence was then subjected to PCR and visualised by agarose gel electrophoresis. Results indicated that both TPRE and water aminosilanised nanoparticles allowed the detection of a $566 \mathrm{bp}$ fragment of the target bacteria 16S rRNA gene. However, it was clear that in the case of the TPRE aminosilanised nanoparticles a much more intense band was obtained (see Figure 2c1). This $16 \mathrm{~S}$ rRNA gene sequence is generic to all bacteria, conserved amongst them and mostly present in multi-copy form. In a second hybrid capture experiment following a similar protocol (see Methods), a 310 bp section of the pfkB-thrS intergenic region of Salmonella enterica was specifically targeted. In this case an oligonucleotide capture sequence was conjugated to the aminosilanised silica-magnetite nanoparticles surfaces which was complementary to a region within that fragment and results are shown in Figure 2c2. It was clear that the nanoparticles which had been aminosilanised via TPRE method permitted the detection of this fragment whereas nanoparticles aminosilanised in water did not. In the latter case a band approximately $400 \mathrm{bp}$ in size was observed on gels, indicating a probable lack of specificity in the process (see Figure $2 \mathrm{c} 2$ ). These results clearly imply that the materials produced via TPRE possessed a significantly greater ability to detect the target 


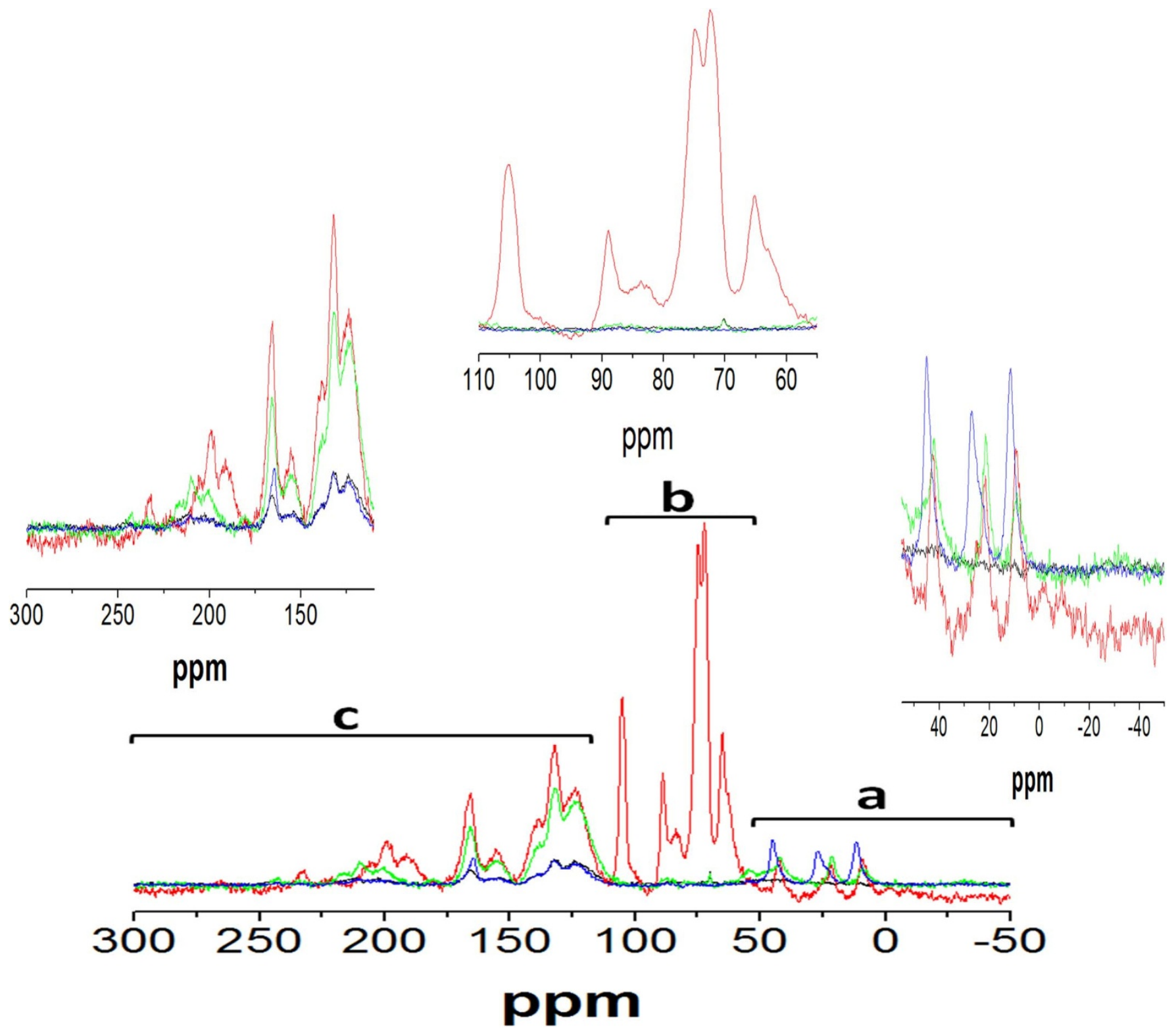

Figure $4 \mid{ }^{13} \mathrm{C}-{ }^{1} \mathrm{H}$ CPMAS NMR spectra of; non-aminosilanised silica nanoparticles (black); condensed/polymerised APTS (blue); unwashed TPRE aminosilanised silica nanoparticles (red); and washed TPRE aminosilanised nanoparticles (green).

sequences in question, confirming the hypothesis that their surface patterning with oligonucleotide was 'better' than that of the materials aminosilanised in water.

\section{Discussion}

In summary we have demonstrated that tri-phasic reverse emulsion (TPRE) is a simple and efficient method for the surface engineering of nanoparticles in suspension where a surface ad-layer of water can control the ordering of nanoparticle surface amine groups during aminosilanisation. The resultant oligonucleotide conjugated nanoparticles functioned as highly efficient bio-sensors. We contend that TPRE would prove useful for any nano or microparticle surface activation/patterning in suspension where an organosilane is involved and a well characterized, high density, effectively monolayer of surface functional groups is desirable.

\section{Methods}

Silica-coated magnetic nanoparticles and model silica nanoparticles synthesis. Silica- magnetic nanoparticles were synthesised by previously reported approaches ${ }^{20,21}$ and diamagnetic silica spheres by the Stober method ${ }^{22}$.
TPRE aminosilanisation of nanoparticles. $150 \mathrm{mg}$ of either core-shell silicamagnetite nanoparticles or diamagnetic silica spheres were collected from aqueous suspension by magnetic separation or centrifugation. $30 \mathrm{ml}$ of toluene and $5 \mathrm{gm}$ of Triton X100 were added and the mixture shaken to form a tri-phasic reverse emulsion (see Movie S1; Supplementary Information). APTS was added to the emulsion to a final concentration of $2 \% \mathrm{w} / \mathrm{v}$ and the mixture allowed to react in a $100 \mathrm{~mL}$ glass reactor fitted with condenser at $50^{\circ} \mathrm{C}$ in an oil bath for $5 \mathrm{hrs}$ with stirring. The nanoparticles were collected by either magnetic separation (superparamagnetic silica-magnetite) or centrifugation (diamagnetic silica), washed with coupling solution $[0.8 \%(\mathrm{v} / \mathrm{v})$ glacial acetic acid in dry methanol] three times and stored at RT in the same solution. Prior to use aminosilanised material was washed with de-ionized water.

Aminosilanisation of nanoparticles in water. This followed the protocol described in reference 12 .

Physical and chemical analysis. Particle sizes in suspension were determined by dynamic light scattering (DLS) using a Malvern Instruments Zetasizer Nano-ZS and Malvern DTS version 5.00 software. Transmission electron micrographs (TEM) of nanoparticles were obtained using a JEOL JEM-1230 electron microscope at $120 \mathrm{kV}$ and samples were placed on carbon coated copper grids (400 mesh, Agar Scientific, UK). ${ }^{29} \mathrm{Si}$ single, ${ }^{29} \mathrm{Si}-{ }^{1} \mathrm{H}$ and ${ }^{13} \mathrm{C}-{ }^{1} \mathrm{H}$ solid state CPMAS NMR spectra were recorded on a JEOL $300 \mathrm{MHz}$ instrument operating at $294 \mathrm{~K}$. Solid materials were carefully compacted into a rotor $\left(6 \mathrm{~mm}\right.$, zirconium oxide) and spun at $6 \mathrm{kHz} .{ }^{29} \mathrm{Si}$ Chemical 
shifts (ppm) were calculated relative to an external standard 2, 2-dimethyl-2silapentane-5-sulfonate (DSS). BET surface area was determined using a Micromeritics ASAP2010 instrument. Surface amine analysis was carried out following a previously published protocol ${ }^{11,12}$. Combustion analyses were performed using a Carlo Erba 1106 combustion elemental analyser.

Model hybrid capture assay. Covalent coupling of single stranded oligonucleotides $\left(5^{\prime}-\mathrm{NH}_{2} \mathrm{dC}_{6} \mathrm{dT}_{25}\right)$ to nanoparticles and DNA hybrid capture of complementary oligonucleotide $\left(5^{\prime}-\mathrm{FdA}_{25}\right)$ experiments were carried out according to a previously published protocol ${ }^{12}$

Oligonucleotide surface functionalised nanoparticles used in the detection of target microbial DNA sequences as particle based bio-sensing. Capture probe design. The oligonucleotide probe sequence for Salmonella enterica was selected from the pfkB-thrS intergenic region (co-ordinates 1501 to 1482, GenBank No. AY064419) using PrimerQuest (available at http://eu.idtdna.com/Scitools/Applications/ Primerquest/). The capture probe (CP) sequence

(5'-ATCCATCTAGCCAACCATTG-3') was checked in silico for specificity using BLAST (Basic Local Alignment Search Tool, available through http://www.ncbi.nlm.nih.gov/BLAST/). In the case of the 16S rRNA gene, two probe sequences were chosen, one specific for Gram positive $(16 \mathrm{~S}+)$ bacteria $16 \mathrm{~S}$ rDNA (5'-GAGGAAGGIGIGGAIGACGT-3') and a second specific for Gram negative (16S-) bacteria 16S rDNA ( $5^{\prime}$-AGICCCGIGAACGTATTCAC- $\left.3^{\prime}\right) .{ }^{23}$ Each probe was terminally modified with a $\mathrm{C} 6$ amino linker and all probes were purchased from Sigma-Proligo (Sigma-Proligo France SAS, Paris, France).

DNA hybrid capture experiments with bacterial genomic DNA. A mixture containing purified genomic DNA from Salmonella enterica (100 ng), Listeria monocytogenes $(100 \mathrm{ng})$ and Shewanella putrefaciens $(100 \mathrm{ng})$, was prepared in a final volume of sterile $0.4 \mathrm{ml}$ lysis buffer ( $50 \mathrm{mM}$ Tris- $\mathrm{HCl} \mathrm{pH}$ 8.0, $50 \mathrm{mM}$ EDTA pH 8.0). The mixture was boiled for 10 min to denature the DNA and immediately cooled in ice. Meanwhile, $50 \mathrm{pmol}$ of oligonucleotide-nanoparticle conjugate were washed with sterile $\mathrm{ddH}_{2} \mathrm{O}$, resuspended in $50 \mu \mathrm{l}$ sterile $\mathrm{ddH}_{2} \mathrm{O}$ and heated $\left(85^{\circ} \mathrm{C}, 5 \mathrm{~min}\right)$ to remove possible oligonucleotide secondary structures. Subsequently, the conjugate, $0.13 \mathrm{ml} 20 \times \mathrm{SSC}(3 \mathrm{M} \mathrm{NaCl}, 0.3 \mathrm{M}$ sodium citrate $\mathrm{pH} 7.0)$ and $20 \mu \mathrm{l} 5 \%$ (w/v) BSA were added to the DNA mixture and the final volume brought to $1 \mathrm{ml}$ using sterile $\mathrm{ddH}_{2} \mathrm{O}$. The resulting mixture was incubated at $40^{\circ} \mathrm{C}$ for 3 hours in a Hybridiser oven (Techne, Cambridge, UK) with end-over-end rotation. After hybridisation the conjugates were collected by magnetic separation, the supernatant discarded and washed twice with $0.2 \mathrm{ml}$ sterile washing buffer $(1 \mathrm{M} \mathrm{NaCl}, 10 \mathrm{mM}$ Tris- $\mathrm{HCl} \mathrm{pH} \mathrm{7.4,1 \textrm {mM }}$ EDTA). Finally the nanoparticles were re-suspended in $20 \mu \mathrm{l}$ of $\mathrm{ddH}_{2} \mathrm{O}$ and $3 \mu \mathrm{l}$ of suspension were used as template in PCR.

PCR. Target DNA capture was confirmed by PCR. In the case of Salmonella enterica, a 310 bp fragment was amplified using primers Sal_f

5' -TTGCCTGAAGCGGTAGCCAG-3' and Sal_r

5'-GGTGACCCATACGCAAGATC-3', whereas in the case of $16 \mathrm{~S}$ rDNA, a 566 bp fragment was amplified using primers $341 \mathrm{f} 5^{\prime}$-GCCTACGGGAGGCAGCAG-3' and 907r 5'-CCGTCAATTCATTTGAGTTT-3' . A typical PCR reaction (final volume of $50 \mu \mathrm{l})$ consisted of: $3 \mu \mathrm{l}$ oligo-nanoparticles suspension as template, $0.2 \mu \mathrm{M}$ each primer, $200 \mu \mathrm{M}$ each deoxynucleotide, $1 \times$ ThermoPol Reaction Buffer $(2 \mathrm{mM}$ $\mathrm{MgSO}_{4}$ final concentration) (New England Biolabs (UK) Ltd, Hitchin, UK), $0.2 \mu \mathrm{g} / \mu \mathrm{l}$ BSA and 2U VentR® DNA Polymerase (New England Biolabs (UK) Ltd). Thermal cycling was carried out in a Progene Thermal Cycler (Techne, Cambridge, UK) and was as follow; $95^{\circ} \mathrm{C}$ for $5 \mathrm{~min}, 50 \mathrm{cycles}$ of $95^{\circ} \mathrm{C}$ for $30 \mathrm{~s}, 60^{\circ} \mathrm{C}$ for $30 \mathrm{~s}, 72^{\circ} \mathrm{C}$ for $1 \mathrm{~min}$. The protocol was completed by a final extension at $72^{\circ} \mathrm{C}$ for $5 \mathrm{~min}$. The expected PCR amplification products were visualised on a $2 \%$ agarose gel (SigmaAldrich Company Ltd, Gillingham, UK) stained with $0.5 \mu \mathrm{g} / \mathrm{ml}$ ethidium bromide.

1. Barth, J. V., Costantini, G. \& Kern, K. Engineering atomic and molecular nanostructures at surfaces. Nature 437, 671-679 (2005)

2. Langer, R. \& Tirrell, D. A. Designing materials for biology and medicine. Nature 428, 487-492 (2004).

3. Maxwell, D. J., Taylor, J. R. \& Nie, S. M. Self-assembled nanoparticle probes for recognition and detection of biomolecules. J. Am. Chem. Soc. 124, 9606-9612 (2002)

4. Nakagawa, T. et al. Fabrication of amino silane-coated microchip for DNA extraction from whole blood. J. Biotechnol. 116, 105-111 (2005)

5. De Feyter, S. Surface patterning from 2D to 3D. Nat. Chem. 3, 14-15 (2011)
6. Kurth, D. G. \& Bein, T. Quantification of the reactivity of 3-aminopropyltriethoxysilane monolayers with the quartz-crystal microbalance. Angew. Chem., Int. Ed. Engl. 31, 336-338 (1992).

7. Kurth, D. G. \& Bein, T. Surface-reactions on thin-layers of silane coupling agents. Langmuir 9, 2965-2973 (1993).

8. Ek, S., Iiskola, E. I. \& Niinisto, L. Atomic layer deposition of amino-functionalized silica surfaces using $\mathrm{N}$-(2-aminoethyl)-3-aminopropyltrimethoxysilane as a silylating agent. J. Phys. Chem. B. 108, 9650-9655 (2004).

9. Gartmann, N., Schutze, C., Ritter, H. \& Bruhwiler, D. The effect of water on the functionalisation of mesoporous silica with 3-aminopropyltriethoxysilane. J. Phys. Chem. Lettt. 1, 379-382 (2010).

10. Natai, A. et al. Pore surface engineering in covalent organic frameworks. Nat. Commun. 2, DOI:10.1038/ncomms 1542 (2011)

11. van de Waterbeemd, M., Sen, T., Biagini, S. \& Bruce, I. J. Surface functionalisation of magnetic nanoparticles: Qunatification of surface to bulk amine density. Micro Nano Lett. 5, 282-285 (2010).

12. Bruce, I. J. \& Sen, T. Surface modification of magnetic nanoparticles with alkoxysilanes and their application in magnetic bioseparations. Langmuir 21, 7029-7035 (2005).

13. Thanh, N. T. K. \& Green, L. A. W. Functionalisation of nanoparticles for biomedical applications. Nano Today 5, 213-230 (2010).

14. Yang, H., Ou, L. W., Wimbrow, A. N., Jiang, X. P. \& Sun, Y. P. Rapid detection of Listeria monocytogenes by nanoparticle-based immunomagnetic separation and real-time PCR. Int. J. Food Microbiol. 118, 132-138 (2007).

15. Amagliani, G. et al. Development of a magnetic capture hybridization-PCR assay for Listeria monocytogenes direct detection in milk samples. J. Appl. Microbiol. 100, 275-383 (2006).

16. Rother, D., Sen, T., East, D. \& Bruce, I. J. Silicon, silica and its surface patterning with alkoxysilanes for nanomedical applications. Nanomedicine 6, 281-300 (2011).

17. Sen, T., Bruce, I. J. \& Mercer, T. Fabrication of novel hierarchically ordered porous magnetic nanocomposites for bio-catalysis. Chem. Commun. 46, 6807-6809 (2010).

18. Moon, J. H., Shin, J. W., Kim, S. Y. \& Park, J. W. Formation of uniform aminosilane thin layers: An imine formation to measure relative surface density of the amine group. Langmuir 12, 4621-4624 (1996).

19. Vanblaaderen, A. \& Vrij, A. Synthesis and Characterization of Monodisperse Colloidal Organo-silica Spheres. J. Colloid. Interf. Sci. 156, 1-18 (1993).

20. Bruce, I. J. et al. Synthesis, characterisation and application of silica-magnetite nanocomposites. J. Magn. Magn. Mater. 284, 145-160 (2004).

21. Sugimoto, T. \& Matijevic, E. Formation of uniform spherical magnetite particles by crystallisation from ferrous hydroxide gels. J. Colloid. Interf. Sci. 74, 227-243 (1980).

22. Stober, W., Fink, A. \& Bohn, E. Controlled growth of monodispersed silica spheres in the micron size range. J. Colloid. Interface. Sci. 26, 62-69 (1968).

23. Horz, H. P., Vianna, M. E., Gomes, B. P. F. A. \& Conrads, G. Evaluation of universal probes and primer sets for assessing total bacterial load in clinical samples: General implications and practical use in endodontic antimicrobial therapy. J. Clin. Microbiol. 43, 5332-5337 (2005).

\section{Acknowledgements}

This work was supported financially by the European Commission under FP6 (NACBO, contract no. 500804). We thank Dr. Andrew Mendham for practical help with NMR experiments.

\section{Authors contributions}

TS and IJB carried out all the experiments reported here and produced the manuscript.

\section{Additional information}

Supplementary information accompanies this paper at http://www.nature.com/ scientificreports

Competing financial interests The authors declare no competing financial interests.

License: This work is licensed under a Creative Common

Attribution-NonCommercial-NoDerivative Works 3.0 Unported License. To view a copy of this license, visit http://creativecommons.org/licenses/by-nc-nd/3.0/

How to cite this article: Sen, T. \& Bruce, I.J. Surface engineering of nanoparticles in suspension for particle based bio-sensing. Sci. Rep. 2, 564; DOI:10.1038/srep00564 (2012) 